\title{
CORRIGENDUM
}

\section{Using two-sex life tables to determine fitness parameters of four Bactrocera species (Diptera: Tephritidae) reared on a semi-artificial diet - CORRIGENDUM}

\author{
W. Jaleel, J. Yin, D. Wang, Y. He, L. Lu and H. Shi \\ doi: 10.1017/S000748531700092X Published by Cambridge University Press, 25 September 2017.
}

The authors apologise for errors in the above paper. These are explained below.

1. Please replace equation (1) with this

$$
\sum_{x=0}^{\infty} e^{-r(x+1)} l_{x} m_{x}=1
$$

2. In heading "Statistical analysis" Please replace first citation with (Chi, 2017; Chi, 1988; Chi \& Liu, 1985).

3. In the Reference list, please correct this reference "Chi, H. \& Team C. (2017) TWOSEX-MSChart: A Computer Program for the Age-Stage, Two-sex Life Table Analysis. National Chung Hsing University, Taichung, Taiwan." With "Chi, H. (2017) TWOSEX-MSChart: A Computer Program for the Age-Stage, Two-sex Life Table Analysis. National Chung Hsing University, Taichung, Taiwan."

4. Please add these two references in references accordingly:

Chi, H. (1988) Life-table analysis incorporating both sexes and variable development rates among individuals. Environmental Entomology 17: 26-34.

Chi, H., and H. Liu. (1985) Two new methods for the study of insect population ecology. Bulletin of the Institute of Zoology, Academia Sinica 24: 225-240.

\section{Reference}

Jaleel, W., Yin, J., Wang, D., He, Y., Lu, L. \& Shi, H. (2018) Using two-sex life tables to determine fitness parameters of four Bactrocera species (Diptera: Tephritidae) reared on a semi-artificial diet. Bulletin of Entomological Research 108, 707-714. 